\title{
Percepciones estudiantiles sobre seguridad universitaria: El caso de la Universidad Nacional de Costa Rica
}

\section{Student perceptions about university security: The case of the National University of Costa Rica}

\section{Marly Yisette Alfaro Salas}

Escuela de Informática y Programa de Gestión Financiera, Comisión Institucional de Teletrabajo, Universidad Nacional, Costa Rica marly.alfaro.salas@una.cr

\section{Luis Diego Salas Ocampo}

Escuela de Relaciones Internacionales y Programa de Innovación metodológica, participación estudiantil y gestión del conocimiento,

Universidad Nacional, Costa Rica

luis.salas.ocampo@una.cr

Recibido: 14/08/2018 • Aceptado: 04/02/2020

\section{RESUMEN}

Este trabajo busca establecer una línea base de la percepción de seguridad del sector estudiantil en la Universidad Nacional de Costa Rica. Trabaja con una muestra de $n=954$ casos del campus Omar Dengo y la sede Benjamín Núñez. Se utilizó un desarrollo propio con escalonamiento Likert de 94 ítems y el alfa de Cronbach de .650. Existen amenazas de seguridad de corte político y cultural que se expresan en desconocimiento profundo de la materia, inexistencia de una relación de comunicación entre oficiales de seguridad de la institución y el sector estudiantil y una baja legitimidad de los funcionarios que laboran en ese campo. Se concluye que la Universidad tiene una ventana de oportunidad importante para ser gestora de la 
seguridad integral universitaria en conjunto con otros actores locales como las comunidades y los negocios.

Palabras Clave: Seguridad, universidad, estudiante, percepción, policía, comunidad.

\section{ABSTRACT}

This work seeks to establish a baseline of the perception of safety of the student sector at the National University of Costa Rica. It works with a sample of $n=954$ cases of the Omar Dengo Campus and the Benjamín Núñez Campus. We used our own development with 94-item Likert staggering and Cronbach's alpha of .650. There are security threats of a political and cultural nature that are expressed in deep ignorance of the matter, lack of a communication relationship between the security officer of the institution and the student sector and a low legitimacy of the officials who work in that field. It is concluded that the university has an important window of opportunity to be an integral university security manager in conjunction with other local actors such as communities and businesses.

Keywords: Security, University, Student, Perception, Police, Community.

\section{INTRODUCCIÓN}

La seguridad vincula actores e intereses de distinta naturaleza. En un país que no cuenta con ejército como Costa Rica, la institución policial reviste una gran importancia, pues su rol es clave para facilitar el cumplimiento integral de derechos de la población, la convivencia y el ejercicio de ciudadanía, además de la protección del territorio y prevención, atención y reacción a la violencia.

Desde 1949, luego de la abolición del ejército, se pensó en un modelo de policía civilista, en el cual la guardia cumpliese labores de un cuerpo de seguridad nacional, junto con tareas de promoción social comunitaria. Este ejercicio no necesariamente fue armónico, ya que este cuerpo vinculó tanto a militares que habían estado en la guerra civil con personeros de la denominada Policía Militar (Ayala, 2008). 
Posteriormente, en los años setentas se creó la Dirección de Inteligencia y Seguridad (DIS) que ha venido funcionando como policía política bajo las órdenes del Ministerio de la Presidencia y desarrollando actividades de inteligencia para el entorno costarricense. Esto marcó un hito en lo que fue, teóricamente, la gestión de la información para el abordaje científico de la criminalidad y el delito (Matul y Dinarte, 2005).

En un ambiente, donde la seguridad es privilegiada a partir de lo preventivo, el conocimiento es visualizado como un movilizador de acciones de anticipación para que el delito no emerja. Por su condición de institución de producción, validación y uso de conocimiento, cualquier universidad tiene dentro del sistema estatal un rol clave en la gestión de la seguridad.

No obstante, las universidades públicas costarricenses han tenido una noción muy sui generis de la seguridad como producto y proceso; sobre todo, del rol de las instituciones encargadas de proveer las condiciones de ejercicio de este derecho. Esto ha quedado particularmente expresado en las posiciones de la Universidad Nacional y la Universidad de Costa Rica sobre el tema de la defensa de la autonomía universitaria (Baudrit, 2014).

A lo largo del tiempo, estas instituciones de educación superior han tenido una visión interesante sobre la cobertura de los servicios policiales costarricenses dentro de sus campus y sedes. Esto, desde una interpretación de su estatus dentro la estructura estatal (donde estas se conciben como instituciones descentralizadas) y de un principio del que gozan como instituciones educativas, tal es el caso de la autonomía universitaria (como instancias descentralizadas tienen autonomía relativa de decisiones y de gobierno de su competencia legal, salvo los fines que la propia ley establece). No obstante, ya la Sala Constitucional del país ha manifestado que, pese a que las instituciones manejan áreas de autonomía, los cuerpos policiales podrán ingresar a los campus cuando se viole la Constitución Política (Votos 4823-93 y 1306-94).

Pese a la claridad de estos Votos, ha sido popular en las universidades el discurso que plantea a los campus como espacios liberados de la competencia del Ministerio de Seguridad Pública (MSP) y, en consecuencia, la idea errónea de que eventualmente es posible hacer cualquier cosa, porque ahí no tienen jurisdicción las autoridades nacionales. 
Como consecuencia, aunque existentes, en la producción nacional, los trabajos sobre seguridad y universidad (Romero, 2009), particularmente en el tema de la percepción de los estudiantes sobre ella estos han sido escasos en Costa Rica.

Poco se ha realizado, también, para que existan acercamientos entre las universidades costarricenses y el Ministerio de Seguridad Pública, de cara a fortalecer las estructuras de conocimientos de ambas instancias, generación de mecanismos de apoyo y gestiones investigativas conjuntas, etc. Entre la reticencia de los primeros y la falta de estructuras institucionalizadas del segundo, la indiferencia ha ganado terreno.

Este trabajo tiene por objetivo general establecer la percepción de seguridad que tienen estudiantes de la Universidad Nacional de Costa Rica en el campus. Se piensa que esto permite desarrollar los primeros puntos de encuentro para la generación de acciones coordinadas y estratégicas en aras de promover la seguridad como derecho, donde la comunidad académica tiene un rol que cumplir.

Como objetivos específicos se plantean los siguientes:

Describir la percepción existente en el estudiantado con respecto a las políticas de seguridad universitaria generadas por la institución y las dinámicas de involucramiento de los actores de la comunidad universitaria sobre este tema.

Valorar el nivel de legitimidad de los actores institucionales universitarios para su involucramiento en la gestión de la seguridad dentro del campus.

Enunciar las tendencias sobre actividades delictivas generadas a lo interno de la Universidad Nacional sobre la base de la percepción estudiantil.

El Programa Innovación metodológica, participación estudiantil y gestión del conocimiento de la Escuela de Relaciones Internacionales de la Universidad Nacional de Costa Rica (UNA) visualiza, en esta dimensión de la realidad, un quehacer fundamental del ejercicio intelectual, en vista de que tanto estudiantes, personal académico y sectores administrativos se encuentran en los campus universitarios. Por lo tanto, lo que se piensa, siente y hace en términos de la seguridad es competencia directa de la universidad. 


\section{Consideraciones teóricas preliminares}

Un campus universitario constituye un sistema integrado donde personas, instituciones y procesos coexisten y persiguen objetivos comunes. Por ello, la respuesta para generar las condiciones de seguridad debe darse a partir de la interacción de estos elementos y tomando como base sus áreas de experticia. Tal y como señala Lópe Mora, el campo de la seguridad se vuelve un elemento que mundialmente las universidades cada vez toman más en serio, sobre todo, en su reflexión intelectual (Lópe Mora, 2011).

Existen particularidades históricas, contextuales y de énfasis en los estudios internacionales que vinculan el tema de la seguridad y la universidad. Algunos han abordado el fenómeno desde el concepto de seguridad disuasiva, entendida como las posibilidades que el ambiente da para que el sujeto delincuente se vea intimidado para cometer el hecho delictivo en el campus universitario (Reina, 2014). Otros (Pérez, Rojas, Cartagena y Cuartas, 2015) se han centrado más bien en la definición de la inseguridad en el campus, donde destacan el uso del concepto desde una perspectiva política operacional para la clasificación, y exclusión de territorios y poblaciones, como consecuencia de la aplicación de estrategias para garantizar o no el disfrute del derecho.

Desde Estados Unidos este tema ha sido abordado desde una perspectiva más reactiva y operacional. Claramente, existe un conjunto de variables que hacen de naturaleza distinta la incursión de la academia norteamericana en el fenómeno. Por ejemplo, algunos trabajos identifican la cuestión del terrorismo como un elemento transversal en los análisis de seguridad universitaria y los campus (Vest, 2002).

Es tal la importancia otorgada para el caso estadounidense, que hoy el propio Ministerio de Educación ha generado una oficina de educación post secundaria que permite, entre otras cosas, obtener distinta información de forma personalizada sobre delitos y otras incidencias en campus de una buena parte de Estados Unidos. Esto lo hacen en consonancia con las Leyes de Divulgación de la Política de Seguridad del Campus, la Ley de Estadísticas de Delitos del Campus y la Ley de Oportunidades de Educación Superior (Us Deparment of Education, 2020). 
No obstante, para el caso costarricense, los estudios sobre percepciones estudiantiles en el campus universitario, ya sea a nivel público o privado, son escasos. Aunque el tema ha sido abordado por las universidades, su énfasis no ha sido el propio quehacer de las instituciones de educación superior en la promoción de este derecho en sus estudiantes. Mucho menos se ha prestado interés por visualizar el cómo percibe el estudiantado las instituciones en este campo.

En América Latina sí existen otros estudios que vinculan la universidad y la seguridad en sus campus universitarios. El alcance que han tenido estos es diverso y no necesariamente responden al tema de las percepciones estudiantiles sobre la seguridad. Por ejemplo, algunos se han centrado en la parte tecnológica de la seguridad en estos lugares y cómo deben crearse dispositivos para poder reportar de manera inmediata los incidentes (Osorio, Pérez y Sánchez, 2018).

Otros trabajos abordan la relación desde el concepto de comunidad, particularmente en la línea de la seguridad situacional, es decir, tratan de eliminar las condiciones que tienen en la infraestructura que favorezcan riesgos de delito o su efectiva aparición (Sánchez, 2013).

No obstante el tema de lo que estudiantes piensan sobre la seguridad, cómo la viven y además cómo administran las opciones para lograr un manejo del entorno más seguro resulta un tema poco abordado, lo que hace este análisis relevante.

Cómo acercarse al fenómeno de la seguridad en las universidades desde la visión país en Costa Rica

La seguridad en Costa Rica, en el marco actual de desarrollo de la estrategia del Ministerio de Seguridad Pública va más allá de los cuerpos policiales. En su doctrina, otros actores tienen un alto nivel de protagonismo $y$, por ello, las universidades son llamadas a generar conocimiento que permita un crecimiento cualitativo de los enfoques, estrategias y metodologías para la participación y la gestión integral de esta. Particularmente mediante el desarrollo de programas preventivos, el MSP ha optado por el trabajo en comunidades fortaleciendo el binomio Policía - comunidad, que en todo el país ha desarrollado un poco más de cinco mil comités de seguridad comunitaria. Adicionalmente, esta instancia ha venido trabajando 
con los comercios mediante formación, lo cual ha generado sistemas preventivos del delito, con una incidencia moderada, pero significativa para fortalecer la participación de estos actores en la gestión de la seguridad (Moreira, 2016).

Otro de los signos que marca un cambio importante en la doctrina de la seguridad en Costa Rica ha sido el esfuerzo sostenido en el tiempo por hacer que los directores regionales de Fuerza Pública, los comisarios y otros roles de mando tengan un nivel de formación académico que les permita poder gestionar adecuadamente la información y hacer gestión policial desde una perspectiva científica (Moya, 2012).

Es claro que a pesar del esfuerzo realizado por la institución policial por dar un salto cualitativo que garantice la incorporación y participación de otros actores en la prevención del delito y la profesionalización de sus cuerpos, la tarea pendiente aún es grande. Por ello, para que la universidad sea un actor significativo en la seguridad de la comunidad donde se inserta, debe repensar sus propios enfoques de actuación y de gestión de la seguridad en sus campus. Esto implica, por un lado, pasar de los abordajes situacionales del hecho delictivo a dar el salto hacia lo preventivo. Por el otro, trascender de las lecturas de vigilancia de instalaciones y perímetros a promover, en su quehacer cotidiano, los derechos de las personas.

Esto plantea un reto de lo que un oficial debe ser a nivel país y hacia eso se han enfilado los esfuerzos de Costa Rica y, particularmente, el Ministerio de Seguridad Pública. Además de conocer metodologías de intervención, técnicas de manejo de situaciones operativas, debe ser capaz de gestionar a los actores y los contextos de forma tal que pueda prevenir el delito.

Para propósitos de este trabajo, se entiende el oficial de seguridad como un actor medular de desarrollo de seguridad en los entornos nacionales y locales y cumple un rol fundamental en el marco de dotar de estabilidad de cualquier sistema social.

Cuando se piensa en las posibilidades operativas de una gestión preventiva de la seguridad desde una perspectiva donde la comunidad participe activamente y donde las universidades son un actor más en el entramado de actores interesados en la gestión y garantía de derechos en este campo, la situación se vuelve compleja. 
El primer lugar, la situación legal de oficiales de las universidades es cualitativamente distinta. En la Universidad Nacional de Costa Rica, aunque este personal es contratado como guardas y existen mecanismos adecuados de selección, lo cierto es que a la hora de implementar medidas represivas o de control, se topan con una serie de elementos legales que convierten su trabajo en una naturaleza sumamente compleja por su condición jurídica como ente de control. Esto es distinto en otras universidades públicas donde, o bien el oficial de seguridad pertenece a alguno de los cuerpos policiales nacionales, o se encuentra contratado con una modalidad de servicio privado.

En Costa Rica, los cuerpos policiales del Estado según el artículo 6 de la Ley General de Policía (Ley 7410,1994 ) son la Guardia Civil, la Guardia de Asistencia Rural, la Policía encargada del control de drogas no autorizadas y de actividades conexas; la Policía de Fronteras, la Policía de Migración y Extranjería, la Policía del Control Fiscal, la Dirección de Seguridad del Estado, la Policía de Tránsito, la Policía Penitenciaria y las demás fuerzas de policía, cuya competencia esté prevista en la ley. Dentro de sus competencias, se encuentra la de aplicación de la fuerza en las situaciones estrictamente necesarias conforme se requiera, en acuerdo con el artículo 10 inciso $d$.

El servicio privado de seguridad en el país se rige por esta misma Ley a partir del artículo 90 y sucesivos. Estos tienen dentro de sus competencias, las labores de protección para quienes contratan sus servicios en el marco de la ética que corresponde a todos los cuerpos policiales.

En el caso de los oficiales de la Universidad Nacional, el problema con su estatus deviene en el hecho de que estos no forman parte de un ente privado de seguridad, son funcionarios públicos, pero no forman parte de ningún cuerpo policial específico como lo establece la Ley General de Policía.

Para propósitos de la institución, según el Manual de Puestos (Talento Humano UNA, 2017), estos funcionarios se encuentran como parte del personal que cubre servicios generales. Los puestos que aparecen asociados en la estructura a las labores de seguridad son los operadores de acceso vehicular, oficiales de seguridad y oficiales supervisores. Es sumamente interesante el hecho de que el enfoque con el cual se ha generado, tanto el desarrollo de las funciones así como los requerimientos educativos y 
legales, suponen que el énfasis del trabajo de estas personas, son las cosas, las instalaciones y los perímetros territoriales de los campus. Ello queda reflejado en los propósitos y los roles de los tres puestos. En el caso de los oficiales de seguridad se indica que estos realizan funciones básicas para garantizar la custodia de los activos, integridad física del personal y bienes de la institución, mientras que para el caso de los supervisores se dice que estos desarrollan actividades operativas con el fin de garantizar la custodia de los activos de la institución y la seguridad de las personas que permanezcan dentro de las instalaciones (Talento Humano UNA, 2017).

Otro de los aspectos que llaman la atención tiene que ver con los requerimientos educativos y legales para el puesto. Tanto para ser oficial como supervisor, basta tener un segundo año de colegio. En relación con los requerimientos legales, se tiene que también se prioriza en términos de seguridad reactiva. Esto, por cuanto para ambos se pide en primera instancia el certificado psicológico de idoneidad mental para la portación de armas de fuego, el cual debe de estar incluido en el Sistema CONTROLPAS (sistema digital para ingreso de los registros de portación, control y seguridad privada a nivel de armas), haber aprobado el examen del curso teórico práctico para portar armas de fuego, impartido por el Departamento. de Control de Armas y Explosivos del Ministerio de Seguridad Pública, y la licencia para portar armas, emitida por el Ministerio de Seguridad Pública .

Las mismas características formales de nombramiento de oficiales y supervisores evidencian el hecho de que la figura en la cual se definen sus tareas es un híbrido entre un colaborador de soporte administrativo con labores de seguridad, están concebidas desde la idea de que hay que reaccionar al delito una vez que se ha cometido, o bien, de que debe trabajarse con las cosas (instalaciones, iluminación y perímetros) para que el delito no suceda.

No obstante, de cara a la necesidad de articular a las instituciones universitarias con el enfoque país de la gestión participativa de la seguridad a nivel preventivo, se hace necesario que los oficiales de la Universidad $\mathrm{Na}$ cional puedan generar procesos de innovación y de gestión de su trabajo donde prioricen la seguridad de las personas sobre la infraestructura y los activos. Esto, ya que en lo que se señala como salvaguardar la integridad física y la seguridad de las personas como aspecto marginal de los propósitos de los puestos de supervisor y oficial, subyace un elemento profundo 
e importante a nivel de competencia, como es ser un actor fundamental en la gestión integral de la seguridad en la comunidad universitaria, lo cual implica, en términos de sus prácticas, pasar de ser un actor de respuesta inmediata ante eventos (enfoque tradicional de la seguridad) a un actor protagónico de desarrollo de estrategias preventivas y consensuadas con los otros actores, para garantizar la seguridad integral (enfoque moderno de corte preventivo). Adicionalmente, por la naturaleza de las incidencias que atiende, debe desarrollar tanto su capacidad física; pero, sobre todo, la intelectual, para poder dar respuesta a la naturaleza de los problemas que enfrenta en el desarrollo normal de su trabajo. Este aspecto, en la actual política de reclutamiento de personal, se vuelve un reto medular.

El oficial de seguridad universitaria es un actor más de la comunidad, quien se construye en el campus universitario. Esta es un sistema integral e integrado, en el que personal académico y administrativo, y estudiantes interactúan diariamente para poder generar conocimiento e incidir en el país a partir de su experticia y sus competencias en la gestión de la seguridad. Sin embargo, la comunidad universitaria se inserta y participa de forma cualitativamente diferente en otros subsistemas que son relativamente cercanos a los campus universitarios y que también tienen injerencia en la prevención del delito, tales como la organización que se genera con los comités de seguridad comunitaria o los subsistemas de seguridad comercial.

Al comprender este hecho, resulta una tarea medular el conceptualizar cómo la universidad puede articularse en el modelo policía-comunidad. En este sentido, se piensa en la existencia de tres subsistemas medulares que interactúan desde la incorporación de la Universidad para prevenir el delito.

Por ello, la seguridad universitaria se visualiza a partir de la necesaria interacción continua y estratégica entre tres sistemas medulares, sobre los cuales el oficial de seguridad de la UNA debe desarrollar mecanismos de gestión (Figura 1). 
Figura 1

Interacción de los subsistemas sobre los cuales debe actuar el oficial de la UNA para la gestión integral de la seguridad

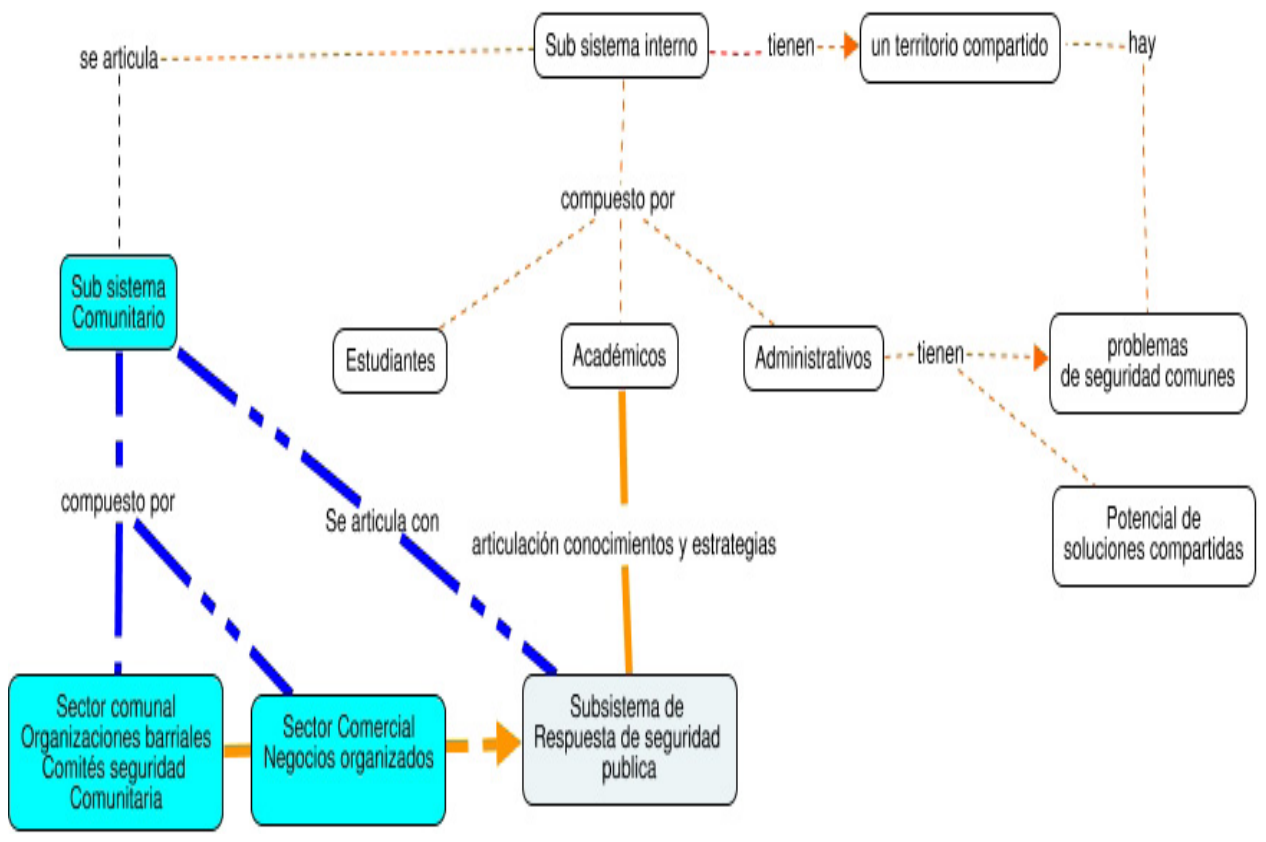

El primer subsistema es el interno, donde el sector administrativo, el estudiantil y el académico, se encuentran en un territorio determinado, comparten un conjunto de prácticas específicas y, además, tienen problemas y potenciales soluciones comunes.

El segundo de estos subsistemas es el comunitario, en las universidades, con dos sectores medulares. El comunal, que constituye tanto redes de personas como de organizaciones barriales, en el que finalmente la Universidad se inserta en este conjunto de relaciones que construyen dinámicas de convivencia. El comercial, uno de los principales interesados en que se generen las condiciones de seguridad necesarias para, por un lado, poder operar y generar ganancias y, por el otro, colaborar con los niveles de desarrollo comunitario. 
Finalmente, el tercer subsistema representado por las instancias de respuesta de seguridad pública, específicamente, la organización que tiene el Ministerio de Seguridad tanto en prevención como en reacción.

En la cotidianidad, los oficiales interactúan con cada uno de estos actores en los contextos. Sin embargo, cómo y para qué interactúan, cuál es la información que generan, cómo incide está en la disposición de recursos y estrategias y, sobre todo, cómo promueven y capitalizan la preocupación de los otros actores de los subsistemas y del propio sistema comunidad universitaria en la gestión integral de la seguridad, son dudas importantes que aún desde las universidades públicas costarricenses no ha sido tematizadas.

\section{Articulación del enfoque planteado a teorías generales sobre seguridad}

Cuando un aparato policial dice que prevendrá el delito, asume teóricamente y a nivel de gestión, un redireccionamiento de los recursos económicos y técnicos al desarrollo de estrategias de información que faciliten la incorporación participativa de otros actores en las tareas de la seguridad, desde los campos particulares de expertiz de cada uno de estos.

La fuerza con la que se realice, desde lo preventivo, la labor de gestión de la información será directamente proporcional a las posibilidades de reducción del índice de criminalidad por parte de las autoridades policiales.

Desde este enfoque, la generación de conocimiento es un quehacer normal de los actores públicos que trabajan con información dentro de un sistema de carácter racional. Esto significa, para la universidad, la generación de modelos, enfoques e instrumentos de seguridad tanto a nivel situacional como en los planos reactivos. Para que esta ventana de oportunidad sea efectiva se deben producir, desde la institución, conocimientos sobre el tema. Es desde este marco donde nace la presente investigación (Salas, 2007).

No obstante, resulta evidente, en América Latina, que la universidad no se ha interesado por abordar la seguridad universitaria. Cuando el tema emerge dentro de la academia, lo hace generando una distancia importante entre los enfoques y los sujetos (Toro, Benítez, Celis \& Bermeo, 2013). 
En este trabajo se entiende la seguridad desde la perspectiva de la securitización:

Esta concepción, es de raíz social-constructivista, niega que este concepto pueda definirse en términos "objetivos", y subraya que las amenazas y la inseguridad en general son construcciones sociales derivadas de nuestro conocimiento y de los discursos que las representan como tales. Se insiste pues en su carácter político y crítico, rechazando de este modo los planteamientos ortodoxos al estudio de la seguridad que se aproximan a ésta como si fuera un campo neutral, y no atienden a los intereses, identidades y valores en juego, en los que tanto los estados como otros actores no estatales-incluyendo grupos de interés económico, o determinadas burocracias gubernamentales_delimitan las agendas políticas. (Escanez, 2015, p. 112)

Aproximarse a la valoración de las percepciones de seguridad de una universidad pública como la UNA, desde el punto de vista estudiantil, facilita, también, el emerger de posturas de actores de diferente naturaleza, con intereses disímiles y sobre los cuales se construyen mecanismos de funcionamiento y de construcción de orden social de carácter inestable. Esto se expresa mediante el discurso o mediante la opinión sobre las condiciones en las que se vive la seguridad.

Desde la perspectiva de la Escuela de Copenhague, se debe hacer el esfuerzo para comprender cómo un determinado asunto se entiende como tema de seguridad y cómo estos, al mutar con lo cotidiano, no son visibilizados como amenaza (Restrepo, 2000).

Desde ahí, es posible visualizar cómo aparece dentro del escenario de las relaciones sociales de los sujetos, un panorama normativo-cultural que hace ver, como normales, elementos de gestión de la seguridad pública que no son ni tematizados ni sometidos al control ordinario de la ciudadanía (Buzan, Waever y Wilde, 1998).

La gestión del riesgo aporta elementos importantes al análisis de la seguridad. Por un lado, contribuye a la politización de este tema como elemento del discurso, pensamiento y acción desde la comunidad académica. Al identificar la probabilidad de que se genere un evento con características 
negativas para una determinada población, logra clarificar las amenazas y las vulnerabilidades, lo que contribuye al mejoramiento preventivo de la gestión de la seguridad. Para que este se genere, ocurre la interacción entre amenazas y vulnerabilidades ( Centro Internacional para la Investigación del Fenómeno de El Niño (ClIFEN), 2017).

La amenaza es un fenómeno histórico que puede desencadenar condiciones peligrosas donde la muerte, lesiones $u$ otros impactos a la salud, al igual que daños a la propiedad, la pérdida de medios de sustento y de servicios, trastornos sociales y económicos, o daños ambientales pueden aparecer. Para el cálculo de esta se visualiza la intensidad (cuán profunda es) y su frecuencia (cuán regularmente emerge) en términos del contexto específico (Estrategia Internacional para la Reducción de Desastres (EIRD), s. f.).

Las vulnerabilidades son características y aspectos específicos de un sistema que lo debilitan ante una amenaza en el marco concreto de sus efectos dañinos. Estas se componen por la exposición (desventaja por posición, ubicación y localización de un determinado sujeto, sistema o comunidad) y la susceptibilidad (grado de fragilidad para amenazar la amenaza). La resiliencia se entiende como la capacidad de los sistemas para resistir, absorber, adaptarse y recuperarse, de manera oportuna y eficaz, de los efectos adversos que una situación no esperada o inevitable pueda generar. Incluye la preservación y la restauración de sus estructuras y funciones básicas, aspecto que compensa las vulnerabilidades (Korc, Hubbard, Suzuki y Jimba, 2016).

Además de los enfoques mencionados, la dimensión situacional de la seguridad opera en la prevención como en la reacción. Para ello, se abordan usualmente en los análisis las instalaciones, capacidades de manejo de la fuerza de los oficiales y aspectos que sean vinculantes con las estrategias específicas de planificación y disposición de recursos dentro del territorio concreto. Este nivel, aunque de respuesta inmediata, no está en contraposición con los enfoques preventivos, ya que introduce distintos niveles de competencia a otros actores además de los policiales.

Por último, el análisis debe tener un factor psicológico y, en el marco de los procesos de securitización, expresar los distintos niveles de intervención y expresión del fenómeno de inseguridad - seguridad de parte de los 
actores. Elementos tales como el manejo temporal y espacial del delito desde el punto de vista de los oficiales, comunidad estudiantil y los propios delincuentes son algunos de los aspectos que privilegia el análisis desde esta perspectiva.

\section{Método}

Este estudio se desarrolla desde una perspectiva exploratoria transversal cuantitativa. Esto, por cuanto al no existir aproximaciones anteriores a la temática, se piensa como relevante el establecimiento de líneas de base para próximas indagaciones. Es un diseño de corte transversal de tipo descriptivo. El proceso de recolección de información se generó durante el segundo semestre del 2016.

\section{Participantes}

Se realizó una encuesta a estudiantes de la Universidad Nacional en los niveles de bachillerato. La muestra fue de $n=954$ participantes de la Sede Omar Dengo y Benjamín Núñez de la Universidad Nacional. La participación fue de carácter voluntario.

El $46 \%$ de la muestra fueron hombres y $54 \%$ mujeres. El $85 \%$ es de la Sede Omar Dengo y el restante $15 \%$ del Campus Benjamín Núñez. La edad promedio de los participantes es de 21 años, adicionalmente, el promedio de familia es de cuatro miembros y el promedio de año de ingreso en la universidad fue el 2014.

\section{Instrumento}

El instrumento es un desarrollo propio con un total de 94 ítems en formato Likert. El alfa de Cronbach es de .650 lo que se considera moderado.

Este presenta una primera sección de preguntas sociodemográficas, aborda variables relacionadas con la edad, sexo, provincia de nacimiento, provincia de residencia, carrera, facultad, actividades generadoras de ingresos, entre otras.

Posteriormente se visualiza un apartado que profundiza la percepción de los estudiantes con respecto a la gestión de políticas de seguridad de la universidad. En esta se profundizan variables relacionadas con el 
conocimiento de estas, la eficacia y eficiencias percibidas, los niveles de conocimiento que manejan los estudiantes sobre seguridad, la percepción con respecto a los académicos como actores de gestión de la seguridad y su valoración del involucramiento de las instancias universitarias en los subsistemas comunales e institucionales de gestión de seguridad.

La tercera sección se centra en la percepción sobre los oficiales, específicamente en una valoración sobre su actuación en términos de protocolos para el abordaje de situaciones de riesgo y de delito, el nivel de satisfacción con respecto a su actuación, las prioridades de atención de estos en su trabajo, etc.

Un cuarto apartado retoma la percepción de los estudiantes en lo referido al presupuesto existente dentro de la Universidad destinado a la seguridad. En este se generan ítems con respecto a cantidad, calidad, destino e impacto en la autopercepción de seguridad.

El quinto apartado del instrumento profundiza la cotidianidad en el campus en términos de la seguridad y el manejo de las personas que cometen delitos. Se retoman tipos de delitos, presencia, incidencia de estos y niveles de participación.

La última sección de la encuesta establece un conjunto de ítems que tienen que ver con la cantidad de infraestructura, su estado y, además, la incidencia de estos en la aparición de delitos en el campus.

Procedimientos de análisis

La forma de operacionalizar técnicamente los niveles teóricos se expresan en la Tabla 1. 
Tabla 1

Marco de concordancia del proceso de investigación sobre seguridad integral universitaria en la UNA

\begin{tabular}{|c|c|c|}
\hline Constructo & Variables & Dimensiones \\
\hline \multirow[t]{2}{*}{ Riesgos } & Amenazas & $\begin{array}{l}\text { Gestión de políticas de seguridad } \\
\text { Prácticas estratégicas de } \\
\text { oficiales de seguridad } \\
\text { Dimensión económica de la } \\
\text { seguridad }\end{array}$ \\
\hline & Vulnerabilidades & $\begin{array}{l}\text { Habituación a la práctica } \\
\text { delincuencial } \\
\text { Calidad de la respuesta reactiva } \\
\text { Conocimiento de alertas } \\
\text { inmediatas } \\
\text { Gestión de información sobre } \\
\text { actos delictivos }\end{array}$ \\
\hline \multirow[t]{3}{*}{ Reacción } & Instalaciones & $\begin{array}{l}\text { Estado de la infraestructura } \\
\text { Manejo de la infraestructura en la } \\
\text { gestión preventiva }\end{array}$ \\
\hline & $\begin{array}{l}\text { Prevención } \\
\text { situacional }\end{array}$ & $\begin{array}{l}\text { Distribución de los recursos } \\
\text { policiales } \\
\text { Acciones de apropiación del } \\
\text { espacio }\end{array}$ \\
\hline & Manejo de la fuerza & Autonomía universitaria \\
\hline \multirow[t]{2}{*}{$\begin{array}{l}\text { Manejo psico } \\
\text { criminológico del } \\
\text { delito }\end{array}$} & $\begin{array}{l}\text { Manejo espacial del } \\
\text { delito }\end{array}$ & $\begin{array}{l}\text { Contextualización del miedo } \\
\text { Recuperación de espacios de } \\
\text { victimización } \\
\text { Dinámicas organizativas } \\
\text { criminales }\end{array}$ \\
\hline & $\begin{array}{l}\text { Manejo temporal del } \\
\text { delito }\end{array}$ & $\begin{array}{l}\text { Sentido de oportunidad } \\
\text { Días de preferencia para el } \\
\text { desarrollo de actividades } \\
\text { delincuenciales }\end{array}$ \\
\hline
\end{tabular}

Nota: Elaboración propia con base en la aplicación de la encuesta de percepciones sobre seguridad universitaria, 2016.

El tratamiento de los datos en función de los objetivos fue el siguiente:

\section{Objetivo específico 1}

Para describir la percepción existente en los estudiantes con respecto a las políticas de seguridad universitaria generadas por la institución y las 
dinámicas de involucramiento de los actores de la comunidad universitaria sobre este tema, se trabajaron, de manera descriptiva, las siguientes variables:

- $\quad$ Existencia de políticas de seguridad en la institución

- Percepción con respecto a la eficiencia de las políticas

- Percepción con respecto a la eficacia de las políticas

- Existencia de relación de comunicación entre el sector estudiantil y los oficiales de seguridad

- $\quad$ Calidad de la información sobre seguridad suministrada por los oficiales del campus

- $\quad$ Percepción con respecto a la labor del académico como actor preventivo en la gestión de la seguridad

- $\quad$ Seguridad y percepción estudiantil

- Percepción de otros actores con respecto a su papel como protagonistas en el sistema nacional de seguridad

- $\quad$ Percepción sobre los niveles de interacción entre el oficial del campus universitario y la fuerza pública.

- Percepción de los estudiantes sobre sus niveles de participación en seguridad universitaria.

- $\quad$ Articulaciones percibidas entre los niveles del sistema universitario de seguridad

\section{Objetivo específico 2}

Con el fin de valorar el nivel de legitimidad de los actores institucionales universitarios para su involucramiento en la gestión de la seguridad dentro del campus, se definió la legitimidad como parte del manejo del poder desde una perspectiva weberiana. Este la comprendía como la posibilidad de imponer la voluntad sobre la conducta ajena (Weber, 1981). Un componente de este es el ejercicio de la dominación que, en el caso del Estado, necesita depositantes de autoridad para que la elección racional de las personas a obedecer el orden sea respetada en los escenarios concretos por depositarios de esta autoridad. Es decir, si no existen representantes de la autoridad concretos en la vida cotidiana de los actores, no podrán hacer ejercicio fáctico del poder y mucho menos de la labor coercitiva. De 
ahí que se decidiera visualizar la relación existente entre la legitimidad y la presencia de oficiales en el campus.

En el tema de seguridad propiamente dicho, es clásica la relación entre presencia de oficiales y percepción de sentirse seguro (Salomón, 2004, p. 7). Por ello se quiso visualizar la relación percibida entre estas variables y los actores de injerencia en el sistema universitario posible en la gestión preventiva. Para ello se trabajó la siguiente hipótesis:

H1: Existe una correlación directa y positiva entre las variables independientes percepción estudiantil con respecto a la cercanía de la relación entre oficiales de seguridad pública y la UNA para prevenir el delito, percepción con respecto a la satisfacción percibida de los oficiales de seguridad de la UNA, nivel de acatamiento de las instrucciones de los oficiales de seguridad por parte de la población estudiantil, autopercepción de protección en virtud de la labor de los oficiales de la UNA y la variable dependiente percepción de suficiencia de oficiales para cubrir todo el campus.

HO: No existe una correlación directa y positiva entre las variables independientes percepción estudiantil con respecto a la cercanía de la relación entre oficiales de seguridad pública y la UNA para prevenir el delito, percepción con respecto a la satisfacción percibida de los oficiales de seguridad de la UNA, nivel de acatamiento de las instrucciones de los oficiales de seguridad por parte de la población estudiantil, autopercepción de protección en virtud de la labor de los oficiales de la UNA y la variable dependiente percepción de suficiencia de oficiales para cubrir todo el campus.

Se trabajó mediante el desarrollo de una prueba de correlación múltiple producto momento de Pearson con un nivel de confianza del $95 \%$.

Adicionalmente se trabajaron, de manera descriptiva, las siguientes variables:

- $\quad$ Percepción con respecto a la cantidad de guardas de seguridad para cubrir el campus universitario.

- $\quad$ Percepción con respecto a que la Universidad Nacional se vincule con el Ministerio de Seguridad Pública y los negocios de la zona para prevenir el delito. 
- Percepción estudiantil con respecto a la dirección que debería tener el presupuesto de la Universidad Nacional destinado a seguridad.

\section{Objetivo específico 3}

En relación con el objetivo tercero orientado a la descripción de las tendencias sobre actividades delictivas generadas a lo interno de la Universidad Nacional sobre la base de la percepción estudiantil, se consideraron para su abordaje las siguientes variables:

- Percepción sobre la frecuencia de realización de actos delictivos en el campus.

- Proveniencia de las personas que ejecutan actos delictivos en el campus.

- $\quad$ Tipos de delitos percibidos que se ejecutan en los campus.

- Percepción sobre importancia de resolver los problemas de seguridad en el campus.

- Percepción estudiantil sobre las reacciones potenciales que tendrían, si fueran víctimas de un delito.

\section{RESULTADOS}

\section{Sobre políticas universitarias e interacción entre los actores}

Para el establecimiento del riesgo, se trabajó primeramente con variables asociadas a la gestión de las políticas universitarias sobre seguridad. Esto, por cuanto las amenazas son de corte histórico, mientras que las vulnerabilidades son de naturaleza coyuntural. Existen aspectos que se provocan a partir de las relaciones de poder, procesos de desigualdad, exclusión o bien el mantenimiento de las situaciones sociales sin cambio alguno, lo cual se institucionaliza y operacionaliza mediante el establecimiento de políticas y de acciones sociales. En el caso de la seguridad, la presencia o no de políticas universitarias de gestión se constituye como una amenaza.

Al ser consultados los estudiantes sobre la percepción de existencia de políticas en este campo, el $50 \%$ indica estar completamente de acuerdo o de acuerdo en que ellas se encuentran presentes. No obstante, al preguntarles a los jóvenes si están de acuerdo con estas, la mitad permanece 
neutral, frente a solamente el $20 \%$ que dice estar con una posición favorable. Además, esa misma proporción indica que las labores que realiza la institución son ineficaces en esta materia.

Un aspecto que incide en la manera en la que la percepción sobre las políticas institucionales de seguridad se construye es la comunicación como práctica estratégica de la gestión de la seguridad. Esto significa que, si un actor con un rol protagónico en el sistema como lo es el oficial, logra bajar contenidos de importancia a los otros (académicos, administrativos, estudiantes) logra niveles de participación de los otros para que la prevención sea posible. No obstante, cuatro de cada diez estudiantes indican que la información preliminar que reciben de parte de los oficiales es de poca utilidad. Al profundizar los datos, es posible detectar una segunda amenaza de corte comunicativo en la gestión y es de tipo cultural.

Los datos permiten apreciar la inexistencia de una relación de comunicación entre el oficial de seguridad de la institución y el sector estudiantil. Solamente 15 de cada 100 estudiantes ven en esto una realidad, mientras que el $50 \%$ de ellos indica la no existencia de esta. En otras palabras, el estudiante es un actor ausente en la gestión de este tema dentro de la institución o, en el mejor de los casos, completamente indiferente. Es posible entender esta amenaza cultural en tanto la labor de salvaguardar la integridad física de los estudiantes y el resto de la comunidad estudiantil en la definición de los términos de su trabajo ocupa un lugar secundario.

Es interesante el hecho de que tampoco el académico es percibido por el estudiante como un actor que haga algo en términos de prevención de situaciones delincuenciales tal y como se evidencia en la Tabla 2. 


\section{Tabla 2}

Percepción estudiantil con respecto a la existencia de una relación de cercanía con el académico para prevenir situaciones delincuenciales en la UNA durante el primer ciclo de 2016

\begin{tabular}{|c|c|c|}
\hline Opciones de respuesta & Frecuencia & Porcentaje \\
\hline $\begin{array}{l}\text { Completamente en } \\
\text { desacuerdo }\end{array}$ & 151 & 15.8 \\
\hline En desacuerdo & 254 & 26.6 \\
\hline Neutral & 319 & 33.4 \\
\hline De acuerdo & 168 & 17.6 \\
\hline $\begin{array}{l}\text { Completamente de } \\
\text { acuerdo }\end{array}$ & 62 & 6.5 \\
\hline Total & 954 & 100.0 \\
\hline
\end{tabular}

Nota: Elaboración propia con base en aplicación de encuesta de percepciones sobre seguridad universitaria, 2016.

Si se suman las frecuencias completamente en desacuerdo y en desacuerdo, se tiene que cuatro de cada diez estudiantes tienen percepciones negativas con respecto al rol del académico en términos de su cercanía para generar acciones de prevención delincuenciales. Si se le agrega el hecho de que tres de cada diez estudiantes permanecen neutrales al valorar el nivel de involucramiento de los profesores, se concluye que es un sector que no es visto con un protagonismo importante. Esto resulta interesante, en vista de que, efectivamente, el tema es completamente ausente dentro de los discursos de las unidades con respecto a la preparación para el ciclo lectivo y también ausente, en la mayoría de los casos, en los contenidos.

El panorama empeora cuando se profundiza en la percepción que tiene el estudiante del oficial de seguridad de la UNA en su interacción con la Fuerza Pública o la policía nacional. En este caso, el $40 \%$ no ven relación alguna, mientras que el $46 \%$ se mantiene completamente neutral a realizar una valoración y solamente un $12 \%$ hace una valoración positiva de las interacciones entre estos entes.

En esta situación es importante determinar, si existen niveles de capacitación en los propios estudiantes con respecto a la seguridad universitaria. El $60 \%$ de la muestra nunca ha recibido una capacitación sobre seguridad 
en el marco de la universidad y solamente el $30 \%$ dice acatar claramente las instrucciones que los responsables le indican.

\section{Sobre la legitimidad de los actores institucionales responsables de la gestión de la seguridad}

Otro elemento que resulta clave para determinar la posibilidad de construcción de una política de seguridad que llegue a las bases universitarias y que permita la operacionalización de mecanismos de gestión a nivel preventivo tiene que ver con la legitimidad de los encargados de administrar la seguridad dentro del campus universitario. Un primer elemento que permite valorar el nivel de legitimidad tiene que ver con la presencia en campus de los oficiales de seguridad en cuanto a su cantidad y suficiencia, como se detalla en la Tabla 3.

\section{Tabla 3}

Percepción estudiantil con respecto a si la cantidad de guardas de seguridad es suficiente para la totalidad del campus en la UNA durante el primer ciclo de 2016

\begin{tabular}{cll}
\hline Opciones de respuesta & Frecuencia & Porcentaje \\
\hline $\begin{array}{lll}\text { Completamente en } \\
\text { desacuerdo }\end{array}$ & 256 & 26.8 \\
En desacuerdo & 320 & 33.5 \\
Neutral & 218 & 22.9 \\
De acuerdo & 126 & 13.2 \\
Completamente de & 33 & 3.5 \\
acuerdo & 1 & .1 \\
No responde & 954 & 100 \\
Total &
\end{tabular}

Nota: Elaboración propia con base en aplicación de encuesta de percepciones sobre seguridad universitaria, 2016.

Cuando los estudiantes perciben que los oficiales son insuficientes en cantidad, teóricamente se presenta la misma sensación que la ciudadanía tiene cuando no existen lo que ellos consideran suficientes policías, tal como se ha logrado observar para el país, se genera una percepción de pérdida 
de la capacidad institucional de los entes represivos del Estado (PNUD, 2005). Con esta situación teórica posible se quiso realizar un análisis en el efecto potencial en la legitimidad, o lo que es igual, en las posibilidades de que la Universidad Nacional realizara con los otros actores una gestión preventiva en esta materia.

Los resultados se evidencian en las correlaciones producto momento Pearson detalladas en la Tabla 4.

\section{Tabla 4}

Correlaciones producto momento Pearson entre el ítem: La cantidad de guardas de seguridad son suficientes para la totalidad del campus y otros ítems en la UNA durante el primer ciclo de 2016

\begin{tabular}{|l|l|l|}
\hline \multicolumn{2}{|l|}{ Ítems correlacionados positivamente } & $\begin{array}{l}\text { La cantidad } \\
\text { de guardas de } \\
\text { seguridad son } \\
\text { suficientes para la } \\
\text { totalidad del campus }\end{array}$ \\
\hline $\begin{array}{l}\text { Existe una importante relación } \\
\text { entre los oficiales de seguridad de } \\
\text { la UNA y la Fuerza Pública para } \\
\text { prevenir acciones delincuenciales }\end{array}$ & Correlación de Pearson & $.337^{* *}$ \\
\hline $\begin{array}{l}\text { La labor de los guardas de } \\
\text { seguridad es satisfactoria }\end{array}$ & Correlación de Pearson & $.486^{* *}$ \\
\hline $\begin{array}{l}\text { Se acatan las indicaciones de } \\
\text { los oficiales de seguridad en la } \\
\text { universidad }\end{array}$ & Correlación de Pearson & $.353^{* *}$ \\
\hline $\begin{array}{l}\text { Me siento muy protegido por los } \\
\text { oficiales de seguridad de la UNA }\end{array}$ & Correlación de Pearson & $.325^{* *}$ \\
\hline
\end{tabular}

**. La correlación es significativa al nivel .01 (bilateral).

Nota: Elaboración propia con base en aplicación de encuesta de percepciones sobre seguridad universitaria, 2016.

Existen correlaciones positivas bajas entre las variables independientes y la percepción con respecto a la suficiencia de la cantidad de oficiales de seguridad para la totalidad del campus. Esto significa que en la medida en que estos aspectos se conviertan en elementos de gestión operativa, se vería reforzada no solamente la imagen del oficial, sino la credibilidad y legitimidad de este en primera instancia como actor depositante de autoridad institucional. 
La prueba también permite valorar el hecho de que, efectivamente, la cantidad de oficiales existentes dentro del campus ayuda o no a fortalecer la credibilidad de la Universidad como actor para garantizar la seguridad de los sujetos.

A partir de estos datos, se comprende por qué solamente tres de cada diez estudiantes indican que tienen la disposición de atender la instrucción generada por un oficial de seguridad dentro de la universidad. Mientras que solamente la mitad de la muestra indica que existe respeto hacia ese funcionario.

Ahora bien, como primer paso, en este trabajo, se consideró, con el sector estudiantil, el rol potencial que le ven a los oficiales de la UNA en cumplimiento de estas labores. Según lo mostrado en la encuesta, sobre la percepción del estudiante en cuanto a que la comunidad e institución se una para prevenir situaciones de delincuencia, solamente un 3,6\% estuvo completamente de acuerdo y un $11,3 \%$ de acuerdo.

El otro componente del subsistema comunitario son los negocios comunes en los alrededores de la institución. Sobre esta vinculación, el sector estudiantil manifestó lo expuesto en la Tabla 5.

Tabla 5

Percepción estudiantil sobre las posibilidades de que la universidad y los negocios organizados por el Ministerio de Seguridad Pública se unan para prevenir situaciones delincuenciales

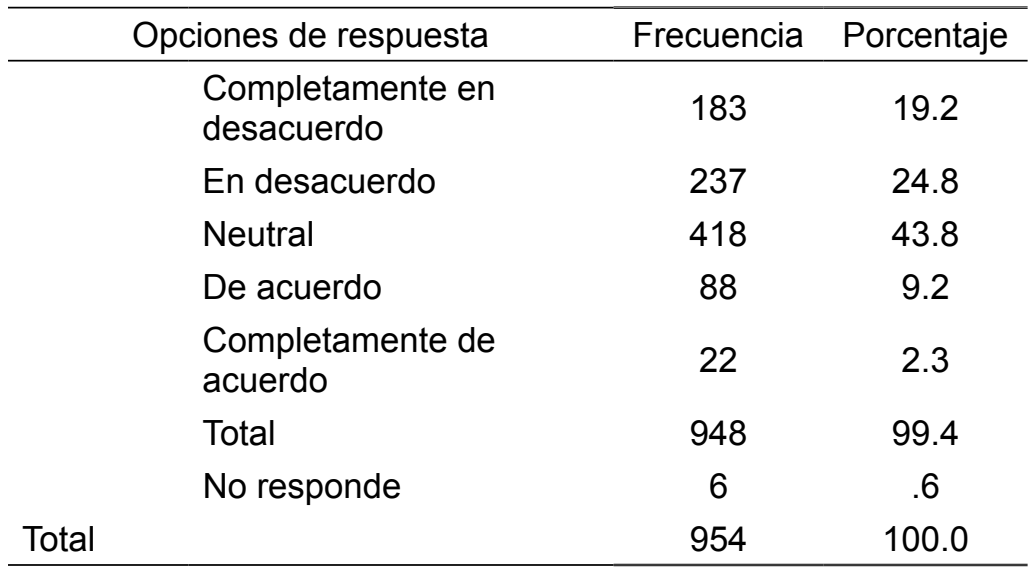

Nota: Elaboración propia con base en aplicación de encuesta de percepciones sobre seguridad universitaria, 2016. 
En este caso, también es más evidente que el estudiante no imagina a la Universidad vinculada con los negocios aledaños a los campus para la prevención del delito, por cuanto solamente un $2,3 \%$ se mostró muy de acuerdo y un 9,2\% de acuerdo; esto reduce en $4 \%$ la percepción de la relación universidad y la comunidad en cuanto a seguridad.

De esto se desprenden varias dudas en relación con lo que significa la labor y proyección social de la universidad en los entornos espaciales más inmediatos donde se ubica. El primer lugar, si la universidad no coordina ni con comunidades ni con negocios a nivel preventivo, con quién sí lo hace. Siendo una instancia en la cual la vinculación con las comunidades a partir de la extensión es parte de su trabajo sustantivo, sería posible imaginar rutas de gestión posibles. Si la respuesta a esto es que no lo hace, las vulnerabilidades que tal ausencia generan para la propia dinámica interna de la gestión de la seguridad en los campus son enormes, ya que la delincuencia suele operar con los principios de desplazamiento del delito (Clarke y Eck, 2003). Según esto, los delincuentes cambian su ubicación en el espacio para evadir las acciones preventivas. En otras palabras, un sistema organizado y coordinado hace que los antisociales dejen las zonas aledañas al campus, porque encontrarán respuesta preventiva.

Adicionalmente, se quiso conocer la percepción de los estudiantes sobre la dirección del presupuesto destinado a seguridad (Tabla 6).

\section{Tabla 6}

Percepción estudiantil sobre la dirección que debería tener el presupuesto destinado a seguridad en la Universidad Nacional de Costa Rica para el primer ciclo del 2016

\begin{tabular}{lcc}
\hline Destino del presupuesto en seguridad & Frecuencia & Porcentaje \\
\hline No Responde & 25 & 2.6 \\
Armas & 67 & 7.0 \\
Capacitación & 393 & 41.2 \\
Incorporación de tecnología & 193 & 20.2 \\
Incorporación de más & 227 & 23.8 \\
oficiales & & \\
No debe invertirse más dinero & 49 & 5.1 \\
en seguridad & 954 & 100.0 \\
Total & 95 \\
\hline
\end{tabular}

Nota: Elaboración propia con base en aplicación de encuesta de percepciones sobre seguridad universitaria, 2016. 
Ocho de cada diez estudiantes consultados no tienen información sobre la inversión de la Universidad en seguridad. Resulta importante visualizar que sí percibe, esta población, una alta necesidad de capacitación, tal y como lo evidencia la Tabla 6. Si se interpreta la ausencia de relación con otros actores y adicionalmente las implicaciones que tiene para un oficial de seguridad estar en una universidad dando sus servicios, es claro que la labor de cuidar instalaciones y reaccionar al delito no resulta suficiente desde la perspectiva estudiantil.

\section{Sobre la actividad delincuencial y campus de la UNA}

Una posible razón mediante la cual se justificaría la ausencia del accionar de la Universidad en esta materia estaría asociada a la ausencia de delito. Dicho en otras palabras, si nada ocurre, por qué habría que actuar. No obstante, al ser consultados los estudiantes sobre la percepción de la ocurrencia de delitos, su existencia queda evidenciada. Para esta medición la frase utilizada fue "se realizan actos delictivos frecuentemente en el campus"y se obtuvieron los siguientes resultados:

\section{Tabla 7}

Percepción estudiantil sobre la realización de actos delictivos frecuentemente en la Universidad Nacional de Costa Rica para el primer ciclo del 2016

\begin{tabular}{|c|c|c|}
\hline Opciones de respuesta & Frecuencia & Porcentaje \\
\hline $\begin{array}{l}\text { Completamente en } \\
\text { desacuerdo }\end{array}$ & 61 & 6.4 \\
\hline En desacuerdo & 134 & 14.0 \\
\hline Neutral & 288 & 30.2 \\
\hline De acuerdo & 334 & 35.0 \\
\hline $\begin{array}{l}\text { Completamente de } \\
\text { acuerdo }\end{array}$ & 132 & 13.8 \\
\hline No responde & 5 & .5 \\
\hline No responde & 954 & 100.0 \\
\hline Total & 954 & 100.0 \\
\hline
\end{tabular}

Nota: Elaboración propia con base en aplicación de encuesta de percepciones sobre seguridad universitaria, 2016. 
Resulta claro que, para los estudiantes, la comisión de delitos se da con cierta frecuencia. Las preguntas que cobran vigencia en este marco tienen que ver tanto con quiénes son los que los ejecutan y cuáles son los de mayor incidencia.

En relación con el primer cuestionamiento, el $30 \%$ indica que los actos delictivos que ocurren en el campo son realizados por personas que no pertenecen a la comunidad estudiantil, es decir, no son académicos, administrativos ni estudiantes. El $50 \%$ prefiere mantenerse neutrales y solamente un $20 \%$ indica que sí son personas que participan de la comunidad universitaria.

En la Tabla 8 es posible apreciar el tipo de delitos que los estudiantes perciben que con mayor regularidad ocurren en el campus universitario.

Tabla 8

Percepción estudiantil sobre el tipo de actos delictivos en la Universidad Nacional de Costa Rica para el primer ciclo del 2016

\begin{tabular}{cll}
\hline Opciones de respuesta & Frecuencia & Porcentaje \\
\hline No responde & 48 & 5.0 \\
Venta droga & 153 & 16.0 \\
Consumo droga & 290 & 30.4 \\
Hurtos & 118 & 12.4 \\
Robos & 267 & 28.0 \\
Vandalismo & 78 & 8.3 \\
Total & 954 & 100.0 \\
\hline
\end{tabular}

Nota: Elaboración propia con base en aplicación de encuesta de percepciones sobre seguridad universitaria, 2016.

Los datos evidencian que desde la visión estudiantil los delitos están relacionados con las drogas tanto a nivel de venta como de consumo y los hurtos. En ambos casos, el entorno del campus podría facilitar la ejecución de este tipo de acciones. Por un lado, al no tener presencia directa la policía dentro de las instalaciones de la Universidad, las dinámicas de venta se facilitan, ya que el oficial dentro del campus no cuenta con el entrenamiento especializado para intervenir este tipo de actividad. En relación con el hurto, fácilmente un estudiante promedio o un académico porta como 
mínimo teléfono celular, computadora, audífonos y otros objetos de valor que pueden ser vendidos en el mercado negro.

Debe destacarse también que el tipo de delitos señalados coinciden con las denuncias que se han colocado en el Organismo de Investigación Judicial para los lugares cercanos a los campus universitarios. Solamente por citar un ejemplo, para el año 2016 en el cantón central de Heredia, se cometieron un total de 1053 delitos de los cuales el $74 \%$ fueron hurtos y asaltos. Por otro lado, el consumo de drogas es especialmente importante en el cantón central de Heredia, que tiene, por ejemplo, en el caso de marihuana, los niveles más altos (IAFA, 2015). Es decir, posiblemente estos podrían estar cumpliendo el rol de rutas potenciales de escape para los delincuentes. En esta misma proporción, se indica que los antisociales tienen ya detectados lugares preferidos para cometer sus delitos (OIJ,2016).

Por ello no impresiona, luego de que a los estudiantes se les ha cuestionado sobre la dinámica delincuencial en la universidad, que vean este tipo de área de gestión como prioritaria acorde con los resultados de la consulta que se traduce en la Tabla 9.

Tabla 9

Percepción estudiantil sobre la importancia de resolver los problemas de seguridad en la Universidad Nacional de Costa Rica para el primer ciclo del 2016

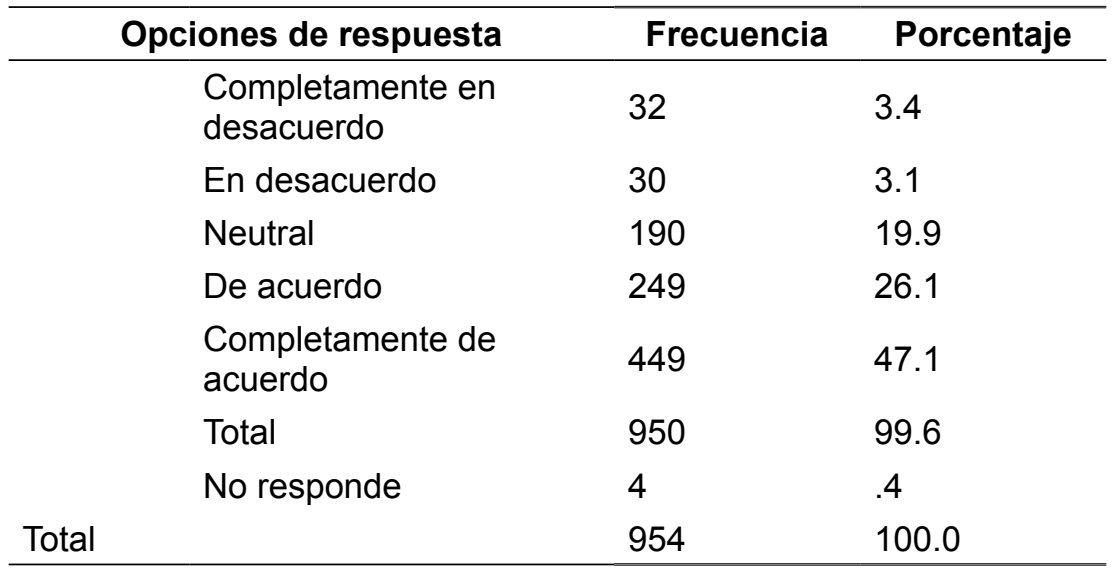

Nota: Elaboración propia con base en aplicación de encuesta de percepciones sobre seguridad universitaria, 2016. 
Esta respuesta es mucho más significativa cuando se observa que el 50 $\%$ de los estudiantes de la muestra dicen no tener ningún tipo de recurso para protegerse individualmente ante una situación delictiva. Esto ocurre en proporciones similares independientemente del tipo del acto (50\% en el caso de asaltos, $45 \%$ en el caso de agresiones, $30 \%$ en casos de violación).

Adicional a la carencia de medidas individuales de protección, solamente el $27 \%$ percibe que podría ser candidato a víctima, frente a un $47 \%$ que indica que bajo ninguna circunstancia estarían en esta situación. No obstante, al colocar a estos estudiantes ante la ejecución de un delito donde estos sean las víctimas, las respuestas ante las posibles reacciones son en extremo interesantes (ver tabla 10).

\section{Tabla 10}

Percepción estudiantil sobre las reacciones potenciales que tendrian si fueran víctimas de un delito en la Universidad Nacional de Costa Rica para el primer ciclo del 2016

\begin{tabular}{llll}
\hline \multicolumn{1}{c}{ Reacciones señaladas } & Frecuencia & Porcentaje \\
\hline & No responde & 13 & 1.4 \\
& Huyo del lugar & 199 & 20.9 \\
& $\begin{array}{l}\text { Me enfrento al } \\
\text { delincuente }\end{array}$ & 134 & 14.0 \\
Válidos & Me someto al delincuente & 124 & 13.0 \\
& Entro en shock & 149 & 15.6 \\
& No sabría qué reacción & 335 & 35.1 \\
tendría & 954 & 100.0 \\
\hline
\end{tabular}

Nota: Elaboración propia con base en aplicación de encuesta de percepciones sobre seguridad universitaria, 2016.

Se evidencia la ausencia de mecanismos en el estudiantado de la Universidad Nacional para enfrentar el delito y para administrar una situación de alto riesgo y estrés. Esto coincide con la escasa preparación país para la gestión de estas situaciones. 


\section{Discusión}

En relación con la percepción de los estudiantes con respecto a las políticas de seguridad y a las dinámicas de involucramiento de actores comunales en el tema, el desconocimiento de estos sobre ambas cosas ha quedado patente.

Un primer elemento que es fundamental para la generación y puesta en marcha de una política de seguridad universitaria, desde la perspectiva de los miembros de la comunidad, es tanto la tematización como la sensibilización sobre este tópico. La clara ausencia de mecanismos para informar sobre las acciones estratégicas que realiza la institución en esta materia, el escaso papel que por definición de puesto tienen los oficiales y supervisores para salvaguardar la integridad de las personas y, además, la escasa información en materia preventiva que estos suministran se convierte en un hallazgo sobre el cual es necesario trabajar. También ha quedado patente que dentro de la labor diaria del oficial del campus, las capacidades de comunicación no son priorizadas en los criterios de contratación, aunque sí se encuentran presentes en los elementos deseables.

Los académicos, al menos desde la perspectiva de los estudiantes, ven el tema como un elemento lejano a sus preocupaciones o aspectos relevantes a profundizar en el curso, en tanto estos no son percibidos como actores en materia preventiva. Es posible que haya consecuencias importantes, si se gestiona una estrategia de involucramiento de la comunidad universitaria en la prevención. Si los académicos no valoran esto como algo importante, no bajarán al nivel de los estudiantes los contenidos que resultan vitales para ponerlos en marcha por parte de la comunidad estudiantil.

La coordinación de la Universidad Nacional con el Ministerio de Seguridad Pública, además de necesaria, es relevante en el contexto actual de la institución. Esto pasa, entonces, por el repensar como institución sus alcances como instancia estratégica en el tema de generación de conocimiento para la toma de decisiones a nivel de operaciones policiales y, además, en su rol como actor estratégico del entramado institucional de las zonas donde se encuentra para hacer gestión preventiva. 
En virtud del segundo objetivo específico de este trabajo, es posible evidenciar que el nivel de legitimidad de los actores institucionales universitarios para su involucramiento en la gestión de la seguridad dentro del campus es bajo.

Queda evidenciado, además, el hecho de que la percepción de presencia del oficial universitario afecta positivamente la percepción de seguridad por parte del estudiante y la legitimidad con la que tanto la administración de la universidad como los otros actores y niveles del sistema pueden operar en materia de gestión preventiva.

El oficial, desde la perspectiva estudiantil, no reviste importancia alguna en términos de su capacidad como actor con credibilidad institucional para marcar el norte en el caso de una situación que atente contra la seguridad del campus.

Aunque debe realizarse una indagación centrada en la percepción de la Universidad Nacional como actor comunitario protagónico en los espacios geográficos donde esta se ubica, al menos en materia de gestión de seguridad, los estudiantes perciben a la institución como un actor ajeno a lo que sucede alrededor y desconectado de los otros actores que gestionan este campo de ejercicio ciudadano.

En el marco actual, desde el punto de vista de seguridad reactiva, la ausencia de protagonismo y de involucramiento sugiere que su campus podría estar siendo utilizado como espacio de resguardo una vez que se comete un hecho delictivo en las comunidades cercanas, esto bajo el principio de desplazamiento delincuencial.

El hecho de que los estudiantes indiquen que el presupuesto universitario de seguridad debería tener un fuerte componente de capacitación, permite apreciar atisbos de un enfoque distinto en su perspectiva de lo que la seguridad debería ser. Esto no es gratuito. Posiblemente las históricas campañas del Ministerio de Seguridad Pública de cara al fortalecimiento del binomio Policía - comunidad han incidido en que estos vean potencialmente una imagen de oficial diferente. Se piensa que esto es una ventana de oportunidad para la gestión. 
Finalmente, en cuanto al tercer objetivo específico del trabajo relacionado con las tendencias sobre actividades delictivas generadas a lo interno de la Universidad Nacional, sobre la base de la percepción estudiantil, es evidente que estos perciben que en el campus universitario sí ocurren delitos similares a los ocurridos alrededor de las comunidades vecinas.

Existe la percepción de que los delitos que se comenten dentro del campus son ejecutados por personas que no pertenecen a la comunidad académica.

La mayor cantidad de delitos, desde la perspectiva estudiantil, se relacionan con drogas y con hurtos. Aunque no se han identificado estudios sobre prevalencia de consumo a lo interno de la Universidad Nacional, sí resulta un tema retador para la profundización. Esto, por cuanto permitirá establecer estrategias de promoción de salud y generación de factores protectores a nivel institucional.

Es claro que el tema de drogas a nivel de consumo y venta, percibido por los estudiantes, podría ser profundizado en términos de capacitación por la sección de vigilancia, por cuanto la ausencia regular de una estrategia de patrullaje y presencia de la fuerza pública a lo interno del campus podría facilitar la venta.

La falta de conocimientos y estrategias de protección ante un hecho delictivo, reconocido por los estudiantes, evidencia la urgencia de que, institucionalmente, se tomen medidas de prevención del delito, pero también se generen estrategias de autoprotección para este sector de la población. Esto siempre desde una perspectiva que privilegie la reducción de riesgos a la vida y a la integridad física.

\section{Recomendaciones}

Para fortalecer la percepción estudiantil sobre seguridad universitaria y, sobre todo, para garantizar que sean legitimados los actores institucionales en su gestión, es necesario que el Consejo Universitario de la institución, dentro de sus temas transversales, pueda ejecutar un análisis concienzudo en materia de seguridad. En este sentido, se hace necesaria una labor de liderazgo de la Vicerrectoría de Administración, instancia sobre la que cae el peso operacional de este tema. 
Además, se hace imperativo que el personal académico se involucre en la gestión preventiva. Es importante que dentro de sus funciones se estipule la relevancia de convertirse en agentes de gestión de información y prevención. En cuanto a lo primero, pues son la cara de la institución en las dinámicas de clase, por lo que se dan cuenta de la incidencia del delito en sus estudiantes y por qué no, también podrían advertir si algunos de estos realizan actividades criminales. También pueden informar a los estudiantes sobre medidas que puedan tomar para disminuir el riesgo. Es, sin duda alguna, un imperativo que este sector sea capacitado.

Se piensa que una manera en la que la Universidad puede hacer un acercamiento con el Ministerio de Seguridad Pública es realizando un levantamiento sobre las capacidades institucionales que esta posee y que pueden tener un impacto positivo tanto en el conocimiento teórico existente sobre esta materia y en lo referido a áreas de incidencia de acciones operativas concretas. Esto pasa también por garantizar un nivel de flexibilización de la acción organizada de programas, proyectos y actividades académicas, de forma tal que puedan adaptar acciones de docencia, investigación y extensión para que aspectos relevantes del tema puedan ser incorporados.

A fin de aumentar el nivel de legitimidad de los actores institucionales universitarios para su involucramiento en la gestión de la seguridad dentro del campus, debe generarse, dentro de la UNA, una campaña de reposicionamiento de la figura del oficial de seguridad. Esto pasa por una valoración importante en términos de la reflexión institucional sobre el aporte que estos oficiales dan en el día a día con su trabajo para lograr un campus donde es posible garantizar la seguridad desde una perspectiva de derechos humanos.

Se piensa que la extensión universitaria podría ser una herramienta medular para que la Universidad Nacional pueda posicionar su identidad marca, sus capacidades y, además, sus conocimientos al servicio de la comunidad. Para esto se requiere de un proceso de reflexión propio sobre sus carencias y lecciones aprendidas en cuanto a cómo se apropia de esta área sustantiva de la acción académica.

Se requiere una importante coordinación con los actores locales en términos de intercambio de información sobre vulnerabilidades, amenazas y riesgos que las comunidades y los negocios aledaños al campus viven, 
para que la Universidad Nacional pueda realizar una investigación específica sobre la incidencia de estos riesgos de seguridad en sus alrededores en la gestión de su propia seguridad.

Es medular que la Sección de Vigilancia de la Vicerrectoría de Administración realice un diagnóstico en sus oficiales sobre las capacidades de gestión preventiva en materia de seguridad. Se piensa que la coordinación estrecha con la Dirección Nacional de Programas Preventivos y la Escuela Nacional de Policía Francisco J. Orlich podría ser una relación posible para dirigir sus esfuerzos de capacitación. Igualmente esto podría fortalecer, potencialmente, la relación con la Dirección Regional de Heredia.

Finalmente, en relación con las tendencias sobre actividades delictivas generadas a lo interno de la Universidad Nacional, desde la perspectiva estudiantil, los delitos que ocurren en el campo no los ejecutan ni estudiantes, ni académicos y tampoco administrativos, lo cual es una ventana de oportunidad para fortalecer la cohesión identitaria con respecto a la seguridad.

Debe generarse una acción de educación, prevención y manejo de situaciones vinculadas con drogas. Para esto podría aprovecharse la existencia de programas institucionales de acondicionamiento físico como ATS y la acción organizada de la Vicerrectoría de Docencia en torno a la participación estudiantil.

Es recomendable también, que la Sección de Seguridad de la UNA pueda levantar un diagnóstico de capacidades entre su propio personal oficial en el tema de drogas, por cuanto, para poder realizar una acción de impacto, es necesario el manejo de conocimientos técnicos en materia de identificación de puntos de venta. Esto supone una estrecha relación e intercambio de información con la Dirección Regional de la Fuerza Pública.

Resulta medular que el sector académico pueda ser capacitado en la promoción de estrategias de autoprotección estudiantil para la prevención del delito. Existen instancias públicas y privadas especializadas que podrían adaptar sus experiencias institucionales previas, en el trabajo con comunidades y otras instituciones, a las esferas del sistema de seguridad universitaria. 


\section{Agradecimientos}

Se deja constancia de agradecimiento al siguiente grupo de estudiantes que colaboraron tanto en el proceso de concepción de la investigación como en la aplicación de instrumentos. Estos fueron parte del grupo del curso Informática y técnicas de investigación, número dos, de la Escuela de Relaciones Internacionales de la Universidad Nacional de Costa Rica durante el primer ciclo del año 2016. Estudiantes que colaboraron: Diego Acuña Picado, Denisse Barboza Flores, Byron Bartels, Luis Bossa, Gabriela Bustos, Kimberley Contreras, Xinia Guzmán, Johel Hernández, Félix Herrera, Élida Jiménez, Anna Manko Konannets, Elia Matarrita, María Miranda, Erick Miranda, Jorge Mora, Larissa Portocarrero, Luis Quirós, José Ruíz, María Toledo, Keilin Torres, Gloriana Valverde, Marcelo Vega, María Vega, Luis Víquez y Brenda Zamora.

\section{Referencias}

Ayala, E. G. (2008). Seguridad y Policía en Costa Rica posterior a la guerra civil de 1948. Diálogos Revista Electrónica, 9, 1710-1729. Recuperado de https://revistas.ucr.ac.cr/index.php/dialogos/article/ download/31309/31015

Baudrit, L. (2014). La autonomía universitaria en la constitución política. Colección Lucem Aspicio, 5. Recuperado de http://www. rectoria. ucr. ac. cr/site/wp-content/uploads/2014/10/Lucem-Aspicio-5. pdf.

Buzan, B., Waever, O., \& Wilde, J. de. (1998). Security: a new framework for analysis / Barry Buzan, Ole Waever, and Jaap de Wilde. Boulder, Colo: Lynne Rienner Pub.

CIIFEN. (2017). Definición de riesgo. Recuperado de http://www.ciifen. org/index.php?option=com_content\&view=category\&layout=blog\&i$\mathrm{d}=84$ \&ltemid=336\&lang $=$ es

Escanez, F. J. V.-M. (2015). Securitización: Agendas de investigación abiertas para el estudio de la seguridad | Securitization: open research agendas for the study of security. Relaciones Internacionales, O(29). Recuperado de http://www.relacionesinternacionales.info/ojs/ 
index.php?journal=Relaciones_Internacionales\&page=article\&op=view\&path $\% 5 B \% 5 \mathrm{D}=652$

Estrategia Internacional para la Reducción de Desastres (EIRD). (2017). Términos principales relativos a la reducción del riesgo de desastres. http://www.eird.org/esp/terminologia-esp.htm

Giraldo, J. (2012). Hablemos de seguridad. Recuperado de http://giroforense.blogspot.com/2012/09/

Instituto sobre Alcoholismo y Farmacodependencia (2015). Prevalencia de consumo reciente, según cantones de Heredia, 2015. Recuperado de http://datosabiertos.iafa.go.cr/visualizations/27988/ prevalencia-de-consumo-reciente-segun-cantones-de-heredia-2015/

Korc, M., Hubbard,S., Suzuki, T., \& Jimba, M.(2016). Salud, resilienciayseguridad humana: Haciala salud para todos. Recuperado de http://iris. paho. org/xmlui/bitstream/handle/123456789/28305/9784889071481_spa. pdf? sequence=1\&isAllowed=y

López Mora, F. (2011). La cultura de seguridad y defensa en el ámbito universitario. Recuperado de: http://helvia.uco.es/handle/10396/11696

Matul, D., \& Dinarte, G. (2005). Enfoques políticos vigentes sobre seguridad ciudadana en Costa Rica. Fundación Friedrich Ebert.

Moreira, J. S. (2016). Incidencia de la seguridad comunitaria en el capital social de barrios urbanos en San José, Costa Rica. URVIO, Revista Latinoamericana de Estudios de Seguridad, 19, 90-110.

Organismo de Investigación Judicial. (2016).Estadísticas policiales del OJI para el año 2016 en el cantón Central de Heredia. Recuperado de https://pjenlinea3.poder-judicial.go.cr/estadisticasoij/

Osorio, R. L., Ortiz, J. A. R., \& Sánchez, L. (2018). Sistema para geolocalizar y reportar incidentes relacionados con la seguridad de usuarios en un campus universitario (a system for geo-localize and report user security-related events in university campus). Pistas Educativas, 4O(130). Recuperado de http://www.itc.mx/ojs/index.php/pistas/ article/view/1740 
Pérez, W. F., Rojas, D. P., Cartagena, L., \& Cuartas, D. (Julio 20l3-junio 20142015). Seguridad e inseguridad en el campus ¿la UdeA es un caso extraordinario? Revista Trabajo Social 18 y 19, , pp. 181-220. Universidad de Antioquía, Antioquía, Colombia. Recuperado de http://www.academia.edu/download/54634841/Perez_2013.pdf

PNUD. (2005). Venciendo el temor y la inseguridad ciudadana. Informe Nacional de Desarrollo Humano Costa Rica. Recuperado de http:// ibdigital.uib.es/greenstone/collect/cd2/index/assoc/pnud0001.dir/ pnud0001.pdf

República de Costa Rica (19 de mayo de 1994). Ley 7410. La Gaceta. Recuperado de http://www.vertic.org/media/National\%20Legislation/ Costa_Rica/CR_Ley_General_de_Policia.pdf

Romero-Pérez, J. E. (2009). El derecho constitucional y la Universidad de Costa Rica: Reflexión en las circunstancias hasta 2009. Revista de Ciencias Jurídicas, 119.

Reina, S. E. (2014). La seguridad disuasiva como método de disminución de riesgos de seguridad física en las instituciones de educación superior en Colombia. Universidad Militar Nueva Granada, Cali Colombia. Recuperado de http://repository.unimilitar.edu.co/ handle/10654/13801

Restrepo, G. A. O. (2000). El aporte de la Escuela de Copenhague a los estudios de seguridad. Revista fuerzas armadas y sociedad, 20(1), 141-162. Recuperado de https://www.academia.edu/download/37460852/EscueladeCopenhague.pdf

Salas, L. D. (2007). El quehacer universitario en la promoción de la seguridad integral. Revista $A B R A, 26(35), 43-54$. Recuperado de https:// www.revistas.una.ac.cr/index.php/abra/article/view/1858

Salomón, L.(2004). El desempeño policial y la percepción ciudadana. Tegucigalpa, Honduras. Programa de Naciones Unidas para el Desarrollo, Universidad de George Town. Recuperado de http://pdba. georgetown.edu/Security/citizensecurity/honduras/documentos/desempeno.pdf 
Sánchez Almeida, M. A. (2013). Los riesgos de accidentes mayores y las condiciones de seguridad en la comunidad universitaria de la Universidad Técnica de Ambato Campus Huachi [Bachelor's thesis, Universidad Técnica de Ambato. Facultad de Ingeniería en Sistemas, Electrónica e Industrial. Maestria en Seguridad e Higiene Industrial y Ambiental]. Recuperado de https://repositorio.uta.edu.ec/ bitstream/123456789/3110/1/Tesis_t786mshi.pdf

Talento Humano Universidad Nacional(2017). Manual de puestos de la Universidad Nacional. Recuperado de https://www.transparencia.una.ac. cr/index.php?option=com_content\&view=article\&id=334\&ltemid=784

Toro, W. F. P., Benítez, L. C., Celis, D. C., \& Bermeo, D. P. R. (2013). Universidad y seguridad. Hechos, situaciones, comunidades. Estudios Políticos, 48, 243-263.

US Depatment of Education. (2020). Campus Safety and Segurity: The tools you need for Campus Safety and Security analysis. Recuperado de https://ope.ed.gov/campussafety/\#/compare/details

Vest, C. (2002). Transparencia, oportunidad y seguridad en las universidades: Un reto nacional. Boston: Discurso a los abogados universitarios y de la Asociación Nacional. Recuperado de http://mit.ocw.universia.net/STS-069/NR/rdonlyres/Science--Technology--and-Society/ STS-069Technology- in-a--Dangerous-WorldFall2002/ B8AE53EDBCFI-4AAB-9AIB-3DCB9D38A437 /O/vestspeech. pdf.

Weber, M (1984). Economía y sociedad. Amorrortu editores. 
